DOI: $10.4274 /$ gulhane.galenos.2020.1049

Gulhane Med J 2021;63:1-7

\title{
New approaches in studying biological mechanisms in psychiatric diseases
}

\section{(1) Şefik Güran}

University of Health Sciences Turkey, Gülhane Faculty of Medicine, Department of Medical Biology, Ankara, Turkey

\section{Date submitted: \\ 11.04.2020 \\ Date accepted: \\ 09.06.2020 \\ Online publication date: \\ 15.03.2021 \\ Corresponding Author: \\ Şefik Güran Prof. MD, University of Health Sciences Turkey, Gülhane \\ Faculty of Medicine, Department of Medical Biology, Ankara, Turkey \\ sefik.guran@sbu.edu.tr}

ORCID:

orcid.org/0000-0002-1398-530X

Presented in: This study was presented to University of Health Sciences, Gülhane Medical Faculty, Department of Psychiatry Clinic as oral presentation in April, 2019.

Keywords: Psychiatric diseases, gene, molecular biology, GWAS, brain bank, animal model.

\begin{abstract}
Special biological markers are needed in psychiatric diseases for understanding the organic etiology. Such kind of biological markers are required in differential diagnosis of psychiatric diseases and in following up of their response to treatment. While the etiology of most psychiatric disorders remains unclear and unknown, there are a lot of supporting evidences that hereditary factors are important in the pathogenesis of psychiatric diseases. Finding the roles of genetic factors in the etiology of diseases probably enhance the understanding of these illnesses better. We can recognize them earlier. Today, we are faced with new scientific developments in this field. Whole genome studies developed in these days have revealed many "hot spot" regions on human genome which could be related to psychiatric disorders. By using new cell culture techniques, we had a chance to have new biological markers in these diseases. Organoids, which resemble original cerebral tissues, were obtained in "in vivo" conditions. Induced pluripotent stem cells were used in organoid techniques for obtaining different cell types. Disease specific animals-genetically modified animals were created with known gene defects of psychiatric diseases in laboratories. New brain banks, which allow us to postmortem studies on human brain, are established in different cities around the world. In the light of the recent developments in cell biology and molecular biology/genetics, this review discusses organic structural findings, taking place in psychiatric disease progression. It is a fact that as the findings increase rapidly in this area, the etiologies of psychiatric diseases will be understood and clarified more easily. By using these technologies, important biological markers will be obtained in the diagnosis. The new treatment protocols can be revealed.
\end{abstract}

\section{Introduction}

General biology and neural system biology in particular have become powerful scientific disciplines in this century. Despite these improvements in biology and neural system biology, no satisfactory pathologic mechanism has been clarified in a psychiatric disease. Achieving a biological mechanism would create a paradigm shift for understanding the psychiatric diseases such as schizophrenia, bipolar disorder, anxiety disorders or obsessive-compulsive disorder $(1,2)$.

Biological markers are needed in psychiatric diseases for understanding the etiology of these diseases. All psychiatric diseases have confusingly similar symptoms, such as long-run sadness, long term irritability, extremely high and low mood in normal conditions, and excessive anxiety or worry $(1,2)$. A biological marker has a potential of revealing the diagnosis of an undiagnosed psychiatric case. Specialists can use these markers in differential diagnosis and in monitoring the achievement of treatment $(3,4)$. Despite the fact that the mechanisms of psychiatric disorders remain obscure, there is strong evidence that hereditary factors are important in the progression of major psychiatric illnesses such as schizophrenia and bipolar disorder (5). Twins studies revealed hereditary predisposition in large series in literature. Big meta-analyses studies in literature presented the importance of inheritance in psychiatric illnesses (6). Genomix (functional genome analyses) and proteomix 
(functional protein analyses) probably point out the significance the hereditary factors. These advances would bring up new approaches to the treatment of psychiatric diseases, an area that has been inert condition for the last thirty years (Table 1) (2).

In this review, the new approaches in cell biology and molecular biology/genetics are mentioned in understanding the pathology of psychiatric diseases. The new culture techniques used in the development of cerebral organoids are explained. Also, the advantages of a genetically engineered mouse model in the uses of experimental studies are tried to be explained. Finally, the roles of brain banks in postmortem studies are discussed (Table 1) (7).

\section{Cell Biology Studies and Findings}

Cell biology studies the structures and functions of cells. New microscopes and staining techniques give an opportunity to see more details within different cells. There are several main subfields in cell biology, including developmental biology and system biology. In developmental biology, cell growth, differentiation and morphogenesis are studied especially in utero conditions. Systems biology is the computational and mathematical analysis of cells. It includes modeling of complex biological systems. System biologists try to explain the interrelationship of gene control mechanisms in entire human genome. On computer simulations, they try to clarify intercellular and extracellular networks (Table 1) (8).
The generation and the maturation of neuronal synapses is important in brain developmant. Neuronal network development is also associated with the elimination process of synaptic structures in brain in gestational period. The elimination of synaptic structure in brain development is known as synaptic pruning. Neuron degeneration (neuronal cell death/neuronal apoptosis) is essential in pruning. Only axon/dendrite degeneration can be seen in a neuron. In neuron degeneration, apoptotic mechanisms are important (9). It is believed that the aims of synaptic pruning are to remove unnecessary neuronal structures from the brain and to reorganize the neuronal/glial structures. At the end of gestation period, simpler neuron structures are obtained in organogenesis period. Dysregulation of pruning in normal brain development seems important in the etiology of autism spectrum disorder (10). In recent years, over proliferation of synapses has been determined after pruning in "in utero" brain development. Such kinds of findings have represented that synaptic reorganizations are important in normal human brain development (11).

Glial cells like neuronal cells also have important role on synaptic pruning in all parts of the nervous system. Glias are the special types of cells in brain structure, which do not generate electrical impulse. Glia cells maintain homeostasis, form myelin, and provide support and protection for neurons. Glia and neuron cells use sets of common signaling pathways in pruning process

\begin{tabular}{|c|c|c|}
\hline Research areas & Main laboratory studies & Main laboratory techniques \\
\hline \multirow[b]{3}{*}{ Genotyping } & Sequencing and bioinformatics findings & $\begin{array}{l}\text { Whole genome analyses and a single gene sequencing techniques w/o } \\
\text { bioinformatics analyses }\end{array}$ \\
\hline & Cell culture studies & $\begin{array}{l}\text { 2-D and 3-D cell culture techniques, induced pluripotent stem cell, and } \\
\text { stem cell studies, organoid studies etc. }\end{array}$ \\
\hline & Animal studies & $\begin{array}{l}\text { Invertebrate models; e.g. C. elegans, fruit fly-drosophila melongester } \\
\text { screens*, } \\
\text { Mouse models for schizophrenia; e.g. disrupted in schizophrenia } \\
1 \text { (DISC1) and } 22 q 11.2 \text { deletion syndrome, and for autism; methyl } \\
\text { CpG binding protein } 2 \text { (MECP2) and 15q11-13 deletion/duplication } \\
\text { syndromes }^{* *} \text {, } \\
\text { Zebrafish }^{* * *}\end{array}$ \\
\hline \multirow{3}{*}{$\begin{array}{l}\text { Molecular biology/ } \\
\text { pathology }\end{array}$} & $\begin{array}{l}\text { Genome-Wide Association Studies } \\
\text { (GWAS), brain tissue studies, etc }\end{array}$ & Metabolomics, genomics, proteomics, transcriptomics etc. \\
\hline & Animal studies & $\begin{array}{l}\text { Brain tissue studies of model animals, obtained intervertebrate, } \\
\text { vertebrate animal models. }\end{array}$ \\
\hline & Human brain tissue studies & Brain tissue studies obtained from brain bank. \\
\hline \multirow{3}{*}{ New therapies } & Chemical screen & Brain tissue studies of model animals and humans. \\
\hline & Cell culture studies & $\begin{array}{l}\text { 2-D and 3-D cell culture techniques, induced pluripotent stem cell, and } \\
\text { stem cell studies, organoid studies etc. }\end{array}$ \\
\hline & Animal studies & Brain tissue studies of model animals \\
\hline \multicolumn{3}{|c|}{$\begin{array}{l}{ }^{*} \text { C. elegans is useful for interference (RNAi) screens. Invertebrate genes exhibit some evolutionary conservation with human genes. Homologues of } \sim 50 \% \text { and } 75 \% \text { c } \\
\text { human disease genes can be identified in C. elegans and D. melanogaster respectively. } \\
{ }^{* *} \text { Mouse is useful for single (or few) gene connections to phenotype, for biomarker studies and for new therapeutic approaches studies. } \\
\text { ***Zebrafish is useful for genetic interactions and variant analysis, for biomarker analyses and for new therapeutic approaches studies. }\end{array}$} \\
\hline
\end{tabular}


(12). Local cytoskeleton disassembly occurs before axon/ dendrite degeneration in pruning. Recent evidences suggest that microtubule disassembly is the local trigger mechanism in pruning $(13,14)$. Dendritic spine formation is a critical process for many synaptic functions in human brain development. The formations and modifications of spines are accepted as vital process for learning and memory abilities of human brain. As known, dendritic spine formation occurs especially in early postnatal period in humans. Like the formation of spines, the removal of dendritic spines is also a normal process in human life. Despite this, we do not know clearly the causes of spine formation and removal in dendrites. The role of dendritic spines' disruption is explained in many neuro-developmental disorders (15). Normally, the strength of synapses can be increased or dicreased in different conditions. Strong action potential is associated with active synapses. Weak synapses are less active and generate weak action potentials. Synaptic plasticity in long term period forms the memory storage conditions in human brain. Long term synaptic depression which is characterized by weak action potentials seems important in the etiology of autism spectrum disorders (16).

As a complex psychiatric disorder, understanding the etiology of schizophrenia is very hard. Schizophrenia pathology is linked to a large number of genes in human genome with highly heritable condition. New technologies improved in cell and molecular biology are tried to be used to find the functions of these genes in disease etiology. Gene abnormalities cause developmental problems in cells such as neuronal migration and axon degeneration (17). Cell movement or migration is the dynamics of cellular processes which include microtubule and adhesion complex dynamics, contraction of actin/myosin filaments (Figure 1) (18). Hockemeyer and Jaenisch (19) have mentioned in a manuscript that schizophrenia patient-derived olfactory cells are more mobile than control-derived cells. Focal adhesion kinase signaling was found as the main factor in the motility of olfactory cells in this experiment.

\section{Stem Cell and Cell Culture Studies}

Stem cells are special cells that can differentiate into other types of cells. They can also divide in self-renewal condition to produce more of the same type of stem cells. Induced pluripotent stem cells (iPS cells or iPSCs) are human generated stem cells, differentiated from terminally differentiated cells. They can divide and differentiate into every other cell type in the body (such as neuron, fat, muscle, and pancreatic cells). Pluripotent stem cells hold promise in the field of regenerative medicine because of these specificities (Table 1) (20).

Cell culture in two dimensions (2D culture) has been routinely used in laboratories worldwide for many years. 2D culture is a simple and easy technique. Despite these advantages, it has very important disadvantages, e.g. it does not show the anatomy or physiology of a tissue. Cell culture in three dimensions (3D culture) is an artificially created environment. In $3 \mathrm{D}$ cell cultures, biological cells are grown and interacted with their surroundings like in tissues (21). Progress in stem cell and iPSC technologies make it possible to use 3D culture systems in laboratory conditions. Today, having an organoid structure is possible using these techniques $(22,23)$. A cerebral organoid is an artificially grown model organ resembling the brain tissues. It can be created for studying a psychiatric disease in a simpler condition. Human brain consists of very complex and heterogeneous structure. The complexity of this tissue makes it difficult to understand how it works in neuroscience (Table 1) (24).

In schizophrenia, a genetically induced vulnerability in brain development was reported. iPSCs obtained from patients with schizophrenia indicate functional problems in neural progenitor cells. Neuronal progenitor cells differentiate into all glial and neuronal cell types, observed in central nerves system. Neural progenitor cells which have functional impairments disturb neocorticogenesis in an organoid model (23). On an organoid in a culture flask, a disorder that has been difficult to study such as microcephaly can be studied also (24).

Autism spectrum disorder is a disorder of neurobiological origin that affects children up to the age of 3. Developmental, cognitive, and behavioral problems occur in an autistic child. Gross motor skills and fine motor skills are also affected due to brain dysfunction. Connectivity problems due to dysfunction in neuronal migration directed by mitochondria are accused of as reliable reason in disease etiology. Intrauterine hyperglycemia and hyperinsulinemia also cause neuronal migration problems which may be important in autism pathology. Today, we know that prolonged neonatal hypoglycemia may cause mitochondrial dysfunction (25). Neuronal migration like neuronal differentiation is a fundamental process occurring in embryonic time period. Today, the effects of many genes on cell migration are put forward in literature. These genes also have similar effects on neural migration. Gene knockout studies in mice have revealed that specific mutations which affect neuron migration proceed severe brain malformations. It seems that such kinds of mutations can cause complex and heterogeneous developmental neuronal migration disorders (26). Cell movement or migration can be achieved by microtubule dynamics, contraction of actin and myosin filaments (Table 1, Figure 1) (18).

\section{Molecular Biology/Genetic Studies and Findings}

Today, we know that a potential genetic predisposition observed in a family is important in the occurrence of many psychiatric disorders such as autism, attention deficithyperactivity disorder, bipolar disorder, major depression and schizophrenia (27). Cytogenetic analyses have revealed many chromosomal alterations on various kinds of psychiatric illnesses in so many 


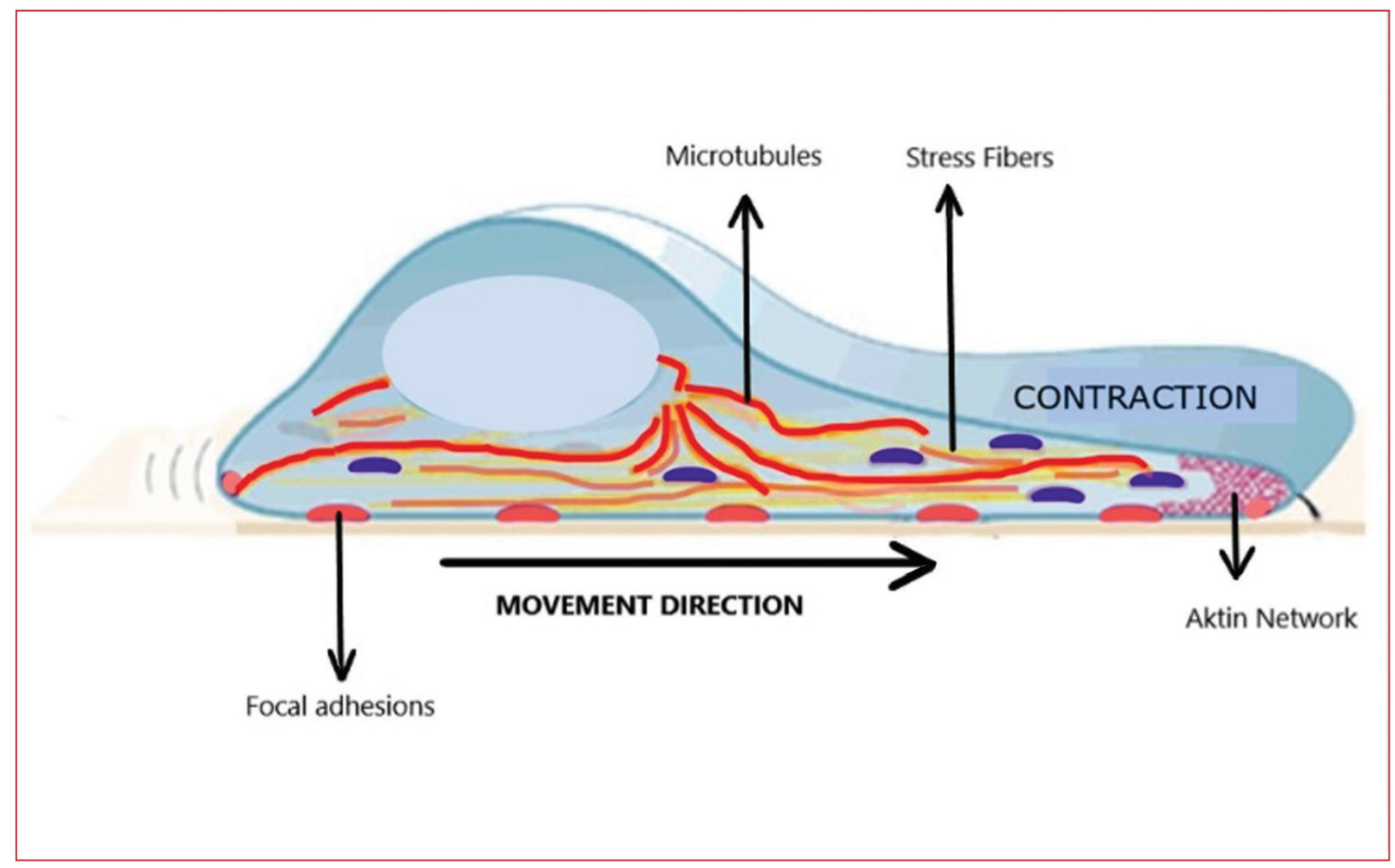

Figure 1. Microtubules and adhesion complexes are important on cell movement and migration. Microtubules and adhesion complexes may probably have roles on the migrations of neuron and glias in embryonic period during the brain development (the figure was modified and drawn again by the author from reference 18)

years. In linkage studies, "hot spots" probably susceptible in psychiatric disorders have been reported (28).

In 1990, a large Scottish family with a $(1 ; 11)(q 42 ; q 14.3)$ translocation was published, which had a significant correlation to specific clinical phenotypes, including schizophrenia and affective disorders (29). Ten years later, Millar et al. (30) described a translocation breakpoint region on chromosome 1q42. The same group identified two novel genes directly disrupted by this translocation ('disrupted in schizophrenia' 1-DISC1 and 'disrupted in schizophrenia' 2-DISC2). By using new techniques, genetically modified mice were obtained in recent years. This type of mouse is a genetically modified mouse in which researchers have inactivated a target gene. They are important animal models for studying the roles of genes which have been sequenced but whose functions have not been determined. Up to now, several DISC1 gene knockout mouse models have been generated for use in experimental studies. All these mouse models display neuro-anatomical and behavioral abnormalities relevant to schizophrenia (Table 1) (31).

In a separate study, the gene DIBD1 has been found to be disrupted by a translocation involving 11q23 chromosome region in a family for bipolar disorder (32). Bipolar disorder is a chronic neuropsychiatric condition characterized by pathological changes in mood of patients from mania to depression. So, bipolar disorders can be accepted as a multifactorial and polygenic genetic disorder. Today, we know that bipolar disorder is the most heritable form of mental illnesses. Despite these, clarifying the hereditary factors in this disease is not easy. Neurodevelopmental factors which affect nervous system development and cell migration were found as altered in bipolar disorders in genome-wide association studies (GWAS) (33). Each GWAS marker appears to confer little risk in bipolar disorder. Even so, common variants together account for $25 \%$ of the heritability of bipolar disorder (34). In these studies, $16 \mathrm{p} 11.2$ gene region had been identified with a higher-risk association. If we found appropriate genetic markers for bipolar disorders, they can be used in the diagnosis of this illness easily (35). Twins studies also have elucidated a high heritability in autistic disorder (36). Large scale meta-analyses in GWAS have reliably identified huge numbers of genetic polymorphisms associated with neuropsychiatric disorders. In most of bipolar disorder, single nucleotide polymorphism (SNPs) located in non-coding genomic regions is correlated with the disease etiology. The molecular importance of SNPs in bipolar disorders is not fully understood. Today, studying the functional properties of disease-associated variants is important for finding the role of these variants in disease etiology in gene expression levels (35). In a manuscript written by Bryzgalov et al. (34), fourteen regulatory SNPs are selected as potential gene locus related to neuropsychiatric disorders. The functions of targeted genes are related to some important processes in cell life cycle such as "posttranscriptional regulation", "neuron differentiation" and "neuron development". On a similar large panel, the psychiatric GWAS consortium found regulatory SNP (rSNP)- targeted genes. This group explained 
the reason of expression differences among patients and control groups in brain region (NRAS-in schizophrenia cohort, CDC25B, DDX21 and NUCKS1-in bipolar disorder cohort) (36). So, new genetic findings observed in psychiatric diseases are important for genetic counseling and genetic testing (Table 1) (37).

\section{Animal Models Obtained by Using New Gene Technologies for Psychiatric Diseases}

Genome/gene editing technology gives opportunity to scientists the ability to change a genome of an organism. In this technology, genetic material can be added, removed, or altered at targeted locations in the genome. Today, generally CRISPRCas9 methodology is used in the laboratories widely because it is faster, cheaper and efficient $(38,39)$. By using CRISPR-Cas9 technology, genetically engineered mouse can be obtained such as DTNBP1 gene disrupted mouse (40). DTNBP1 gene encodes a protein that plays a role in organelle (e.g. lysosome) biogenesis in a cell. Also, this protein has been explained as a causative factor in schizophrenia etiology. We know that it regulates prefrontal brain functions in fetal and adult period. DTNBP1 gene expresses in the dorso-lateral prefrontal cortex and hippocampus. Decreased levels of dysbindin-1 mRNA were found in brain tissues in schizophrenia cases. Scientists claim a strong connection among dysbindin-1 function and schizophrenia pathology (41). In a genetically engineered mouse, which had functionally disturbed dysbindin-1 gene, similar behavioral activities can be observed like in schizophrenic patients. Huang et al. (42) found that this gene has important roles for in the regulations of amygdala functions (Table 1 ).

Reelin protein is located in the extracellular matrix. It is involved in the development of cortical neural connectivity at embryonic phase. Reelin protein generally regulates synaptic plasticity of neurons at postnatal stages. Epigenetic changes in reelin gene in schizophrenic patients probably cause an inhibition in reelin expression in human brain $(43,44)$. Proper cortical development is important in brain structure. In reelin gene mutant mouse (named as a reeler mouse), dysfunction of cortical region was found. The absence of reelin expression in genetically engineered mouse caused migration defects in neurons and developmental defects in dendrites (45).

Injection of human progenitor cells into an immunodeficient mouse gives chance to have "humanized mouse". By using this technology, it can be possible to obtain a brain in the mouse which has "human-like" neuronal structures (46). Capps explained in a manuscript that oligodendrocyte and astrocyte development was found as altered in schizophrenia in chimeric mice experiments. Also, cell-cell interactions were found as important in the pathogenesis of early-onset schizophrenia (47).

Zebra fish represents a good model among complexity and simplicity of system. Studying on zebra fish gives us a good opportunity for finding the mechanisms of brain functions in brain illnesses. Zebra fish can be used as a model for finding the effects of psychotic drugs. Fear and anxiety problems can be analyzed with zebra fish studies easily (Table 1) (48).

\section{Establishing Brain Banks for Postmortem Brain Studies}

For postmortem brain studies, storing and banking of brain tissue samples is an important step. Generally, the brain tissues are stored in liquid nitrogen at $-195^{\circ} \mathrm{C}$ for a long time. Brain banks are given an opportunity to research on the etiologies of different psychiatric diseases. The recent advances in neuroimaging and molecular biology increased the interests to human brain studies in these days (Table 1) (49).

Generally, brain banks collect human brain tissues from donors who had neurological and/or psychiatric disorders. Also, normal human brain tissues are stored in brain banks obtained from healthy donors. The brain tissues stored in brain banks are shared to other scientific researchers around the world. In this way, experiments about understanding the molecular mechanisms in psychiatric diseases can be done on brain samples in all laboratories (50). GWAS are achieving huge successes in identifying disease-associated variants in specific human brain tissues as seen in data in Psychiatric Genomics Consortium (PGC; http://www.med.unc.edu/pgc) (36). The disease-associated variants in psychiatric disease etiology are generally SNPs located in non-coding regions. The effect of each SNP seems in small size in disease etiology. Today, the scientists believe that the cumulative effects of SNPs are stimulated the initiation factors in psychiatric diseases. So, the interpretation of functions in disease associated gene variants can be hidden. In population-based human variation studies, DNA methylation and histone modification were found as important. Gene variant rs199347 is located on an non coding intron region in a protein coding gene named as GPNMB (Glycoprotein Nmb). This variant affects the expression of GPNMB only in human brain. We know that the effect of variant rs199347 is associated with Parkinson disease etiology. This kind of effect in rs199347 variant can not be observed in other tissues in organisms (51). Such kinds of findings represent that the human brain tissues obtained from brain banks can be used in comprehensive molecular studies for advanced researches in neuropsychiatric disorders (50). For that reason, the achievement of brain bank projects is generally dependent on these kinds of complex studies. First of all, the patients and their families should be convinced in the donation of the patient's tissues in postmortem period. And then, the cooperation among scientists and clinicians should be established for more complex studies (49).

\section{Conclusion}

So, developments in cell biology and molecular biology/ genetics techniques reveal new approaches in understanding of 
etiology in psychiatric diseases. The improvements may affect the diagnosis and treatment of these cases in immediate future.

\section{Ethics}

Peer-review: Externally peer-reviewed.

Financial Disclosure: The author declared that this study received no financial support.

\section{References}

1. Etkin A, Pittenger C, Polan HJ, Kandel ER. Toward a neurobiology of psychotherapy: basic science and clinical applications. J Neuropsychiatry Clin Neurosci. 2005;17:145-158.

2. Sachdev PS, Mohan A. Neuropsychiatry: where are we and where do we go from here? Mens Sana Monogr. 2013;11:4-15.

3. Jiménez JP, Botto A, Herrera L, et al. Psychotherapy and genetic neuroscience: An emerging dialog. Front Genet. 2018;9:257.

4. Joyce EM. Organic psychosis: The pathobiology and treatment of delusions. CNS Neurosci Ther. 2018;24:598603.

5. Maclntyre DJ, Blackwood DH, Porteous DJ, Pickard BS, Muir WJ. Chromosomal abnormalities and mental illness. Mol Psychiatry. 2003;8:275-287.

6. Serretti A. Genetics and pharmacogenetics of mood disorders. Psychiatr Pol. 2017;51:197-203.

7. McCammon JM, Sive H. Challenges in understanding psychiatric disorders and developing therapeutics: a role for zebrafish. Dis Model Mech. 2015;8:647-656.

8. Adams JU. Genetics: Big hopes for big data. Nature. 2015;527:108-109.

9. Geden MJ, Romero SE, Deshmukh M. Apoptosis versus axon pruning: Molecular intersection of two distinct pathways for axon degeneration. Neurosci Res. 2019;139:3-8.

10. Johnson MB, Stevens B. Pruning hypothesis comes of age. Nature. 2018;554:438-439.

11. Inta D, Lang UE, Borgwardt S, Meyer-Lindenberg A, Gass P. Microglia Activation and Schizophrenia: Lessons From the Effects of Minocycline on Postnatal Neurogenesis, Neuronal Survival and Synaptic Pruning. Schizophr Bull. 2017;43:493-496.

12. Neniskyte U, Gross CT. Errant gardeners: glial-celldependent synaptic pruning and neurodevelopmental disorders. Nat Rev Neurosci. 2017;18:658-670.

13. Rumpf S, Wolterhoff $\mathrm{N}$, Herzmann S. Functions of Microtubule Disassembly during Neurite Pruning. Trends Cell Biol. 2019;29:291-297.

14. Eaton BA, Fetter RD, Davis GW. Dynactin is necessary for synapse stabilization. Neuron. 2002;34:729-741.

15. Lieberman OJ, McGuirt AF, Tang G, Sulzer D. Roles for neuronal and glial autophagy in synaptic pruning during development. Neurobiol Dis. 2019;122:49-63.
16. Hansel C. Deregulation of synaptic plasticity in autism. Neurosci Lett. 2019;688:58-61.

17. Tee JY, Sutharsan R, Fan Y, Mackay-Sim A. Cell migration in schizophrenia: Patient-derived cells do not regulate motility in response to extracellular matrix. Mol Cell Neurosci. 2017;80:111-122.

18. Horwitz AR, Parsons JT. Cell migration--movin' on. Science. 1999:286:1102-1103.

19. Hockemeyer D, Jaenisch R. Induced pluripotent stem cells meet genome editing. Cell Stem Cell. 2016;18:573-586.

20. Haycock JW. 3D cell culture: a review of current approaches and techniques. Methods Mol Biol. 2011;695:1-15.

21. Çağlayan ES, Güran Ş. Importance of Myc-related microRNAs in induced pluripotency. Cell Biol Int. 2015;39:987-994.

22. Hoffmann A, Ziller M, Spengler D. Focus on Causality in ESC/iPSC-Based Modeling of Psychiatric Disorders. Cells. 2020;9:366.

23. Di Lullo E, Kriegstein AR. The use of brain organoids to investigate neural development and disease. Nat Rev Neurosci. 2017;18:573-584.

24. Nayler SP, Becker EBE. The Use of Stem Cell-Derived Neurons for Understanding Development and Disease of the Cerebellum. Front Neurosci. 2018;12:646.

25. Hoirisch-Clapauch S, Nardi AE. Autism spectrum disorders: let's talk about glucose? Transl Psychiatry. 2019;9:51.

26. Pérez-Brangulí F, Buchsbaum IY, Pozner T, et al. Human SPG11 cerebral organoids reveal cortical neurogenesis impairment. Hum Mol Genet. 2019;28:961-971.

27. Gordovez FJA, McMahon FJ. The genetics of bipolar disorder. Mol Psychiatry. 2020;25:544-559.

28. Preece RL, Han SYS, Bahn S. Proteomic approaches to identify blood-based biomarkers for depression and bipolar disorders. Expert Rev Proteomics. 2018;15:325-340.

29. St Clair D, Blackwood D, Muir W, et al. Association within a family of a balanced autosomal translocation with major mental illness. Lancet. 1990;336:13-16.

30. Millar JK, Wilson-Annan JC, Anderson S, et al. Disruption of two novel genes by a translocation co-segregating with schizophrenia. Hum Mol Genet. 2000;9:1415-1423.

31. Cash-Padgett T, Jaaro-Peled H. DISC1 mouse models as a tool to decipher gene-environment interactions in psychiatric disorders. Front Behav Neurosci. 2013;7:113.

32. Baysal BE, Willett-Brozick JE, Badner JA, et al. A mannosyltransferase gene at $11 \mathrm{q} 23$ is disrupted by a translocation breakpoint that co-segregates with bipolar affective disorder in a small family. Neurogenetics. 2002;4:43-53.

33. O'Shea KS, McInnis MG. Neurodevelopmental origins of bipolar disorder: iPSC models. Mol Cell Neurosci. 2016;73:63-83.

34. Bryzgalov LO, Korbolina EE, Brusentsov II, Leberfarb EY, Bondar NP, Merkulova TI. Novel functional variants at the 
GWAS-implicated loci might confer risk to major depressive disorder, bipolar affective disorder and schizophrenia. BMC Neurosci. 2018;19(Suppl 1):22.

35. Vornholt E, Luo D, Qiu W, et al. Postmortem brain tissue as an underutilized resource to study the molecular pathology of neuropsychiatric disorders across different ethnic populations. Neurosci Biobehav Rev. 2019;102:195-207.

36. Sullivan PF. The psychiatric GWAS consortium: big science comes to psychiatry. Neuron. 2010;68:182-186.

37. Freitag CM, Staal W, Klauck SM, Duketis E, Waltes R. Genetics of autistic disorders: review and clinical implications. Eur Child Adolesc Psychiatry. 2010;19:169178.

38. Komor AC, Badran AH, Liu DR. CRISPR-Based technologies for the manipulation of eukaryotic genomes. Cell. 2017;169:559.

39. Güran Ş, Çoban ZD. The use of induced pluripotent stem cells in medicine. Erciyes Med J. 2012;34:184-187.

40. Singh M, Murriel CL, Johnson L. Genetically engineered mouse models: closing the gap between preclinical data and trial outcomes. Cancer Res. 2012;72:2695-2700.

41. Wang H, Xu J, Lazarovici P, Zheng W. Dysbindin-1 Involvement in the Etiology of Schizophrenia. Int J Mol Sci. 2017;18:2044.

42. Huang CCY, Muszynski KJ, Bolshakov VY, Balu DT. Deletion of Dtnbp1 in mice impairs threat memory consolidation and is associated with enhanced inhibitory drive in the amygdala. Transl Psychiatry. 2019;9:132.

43. Negrón-Oyarzo I, Lara-Vásquez A, Palacios-García I, Fuentealba P, Aboitiz F. Schizophrenia and reelin: a model based on prenatal stress to study epigenetics, brain development and behavior. Biol Res. 2016;49:16.

44. Lossi L, Castagna C, Granato A, Merighi A. The Reeler Mouse: A Translational Model of Human Neurological Conditions, or Simply a Good Tool for Better Understanding Neurodevelopment? J Clin Med. 2019;8:2088.

45. D'Arcangelo G. Reelin mouse mutants as models of cortical development disorders. Epilepsy Behav. 2006;8:81-90.

46. Perrier-Groult E, Pérès E, Pasdeloup M, Gazzolo L, Duc Dodon M, Mallein-Gerin F. Evaluation of the biocompatibility and stability of allogeneic tissue-engineered cartilage in humanized mice. PLoS One. 2019;14:e0217183.

47. Capps B. Do Chimeras Have Minds? Camb Q Healthc Ethics. 2017;26:577-591.

48. Shams S, Rihel J, Ortiz JG, Gerlai R. The zebrafish as a promising tool for modeling human brain disorders: a review based upon an IBNS Symposium. Neurosci Biobehav Rev. 2018;85:176-190.

49. Iritani S, Habuchi C, Sekiguchi H, Torii Y. Brain research and clinical psychiatry: establishment of a psychiatry brain bank in Japan. Nagoya J Med Sci. 2018;80:309-315.

50. Wang L, Xia Y, Chen Y, et al. Brain banks spur new frontiers in neuropsychiatric research and strategies for analysis and validation. Genomics Proteomics Bioinformatics. 2019;17:402-414.

51. Murthy MN, Blauwendraat C, Guelfi S, et al. Increased brain expression of GPNMB is associated with genome wide significant risk for Parkinson's disease on chromosome 7p15.3. Neurogenetics. 2017;18:121-133. 